Témoigner Témoigner. Entre histoire et mémoire

Getuigen Revue pluridisciplinaire de la Fondation Auschwitz

$124 \mid 2017$

La musique dans les camps

\title{
Les camps d'internement juifs dans la France de la zone libre (1940-1942) : Territoires d'une tragédie et lieux de mémoire vivant
} André-Louis Sanguin

\section{(2) OpenEdition}

Journals

Édition électronique

URL : https://journals.openedition.org/temoigner/5809

ISSN : 2506-6390

Éditeur :

Éditions du Centre d'études et de documentation Mémoire d'Auschwitz, Éditions Kimé

Édition imprimée

Date de publication : 2 avril 2017

Pagination : 155-171

ISBN : 978-2-930953-00-7

ISSN : 2031-4183

\section{Référence électronique}

André-Louis Sanguin, « Les camps d'internement juifs dans la France de la zone libre (1940-1942):

Territoires d'une tragédie et lieux de mémoire vivant », Témoigner. Entre histoire et mémoire [En ligne],

124 | 2017, mis en ligne le 30 novembre 2021, consulté le 01 décembre 2021. URL : http://

journals.openedition.org/temoigner/5809 


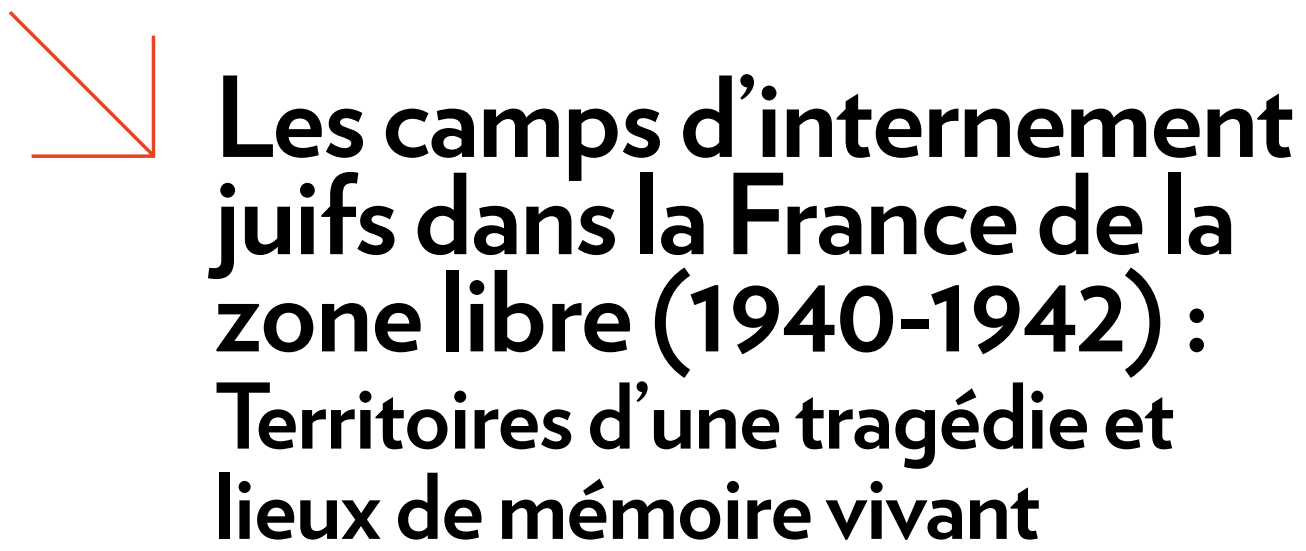

$\rightarrow$ Par André-Louis Sanguin Université de Paris-Sorbonne

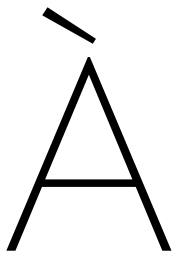

partir de 1933 et de l'instauration de la dictature nazie en Allemagne, des Juifs allemands commencèrent à fuir leur pays devant la montée orchestrée du racisme, consécutive à l'incendie du Reichstag (27 février 1933). La promulgation des lois raciales de 1935 contribua à accélérer ce mouvement. Le gouvernement français ouvrit toutes grandes les portes de son territoire à ces réfugiés politiques d'un genre nouveau. Dans la foulée de l'Anschluss (mars 1938) et des Accords de Munich (septembre 1938), ce furent les Juifs autrichiens et tchécoslovaques qui vinrent grossir ce flux. À cette époque, on dénombrait environ 320000 Juifs en France dont 160000 étaient des étrangers. Or, la population juive s'élevait à 50000 personnes en 1914, mais pendant les années 1920-1930, beaucoup de Juifs avaient immigré en France depuis la Pologne, la Roumanie et l'URSS (Poznanski, 2005).

\section{LA TROISIÈME RÉPUBLIQUE FINISSANTE ET LA CRÉATION DES CAMPS D'INTERNEMENT}

Au début de l'année 1939 s’achevait la guerre civile en Espagne avec la victoire des forces nationalistes de Franco. Barcelone était prise en janvier 1939 et ce qui restait de l'armée de la République espagnole se replia vers les Pyrénées orientales et la frontière française. Elle était accompagnée d'enfants, de femmes, de vieillards fuyant dans la peur et le désordre. Devant l'évidence d'un afflux massif de réfugiés politiques, le Gouvernement Daladier fit construire en trois semaines les camps de Gurs et d'Agde. Le camp du Vernet existait depuis 1918 tandis que celui de Rivesaltes avait été édifié en 1938 pour des usages militaires. Entre février et avril 1939, 450000 Républicains espagnols reculaient massivement et franchissaient la frontière française entre Bourg-Madame et Cerbère. Cela passa à l'histoire sous le nom 
de Retirada (la retraite). En sus des plages d'Argelès, du Barcarès et de Saint-Cyprien, ils furent internés dans les camps de Gurs, du Vernet, de Rivesaltes et d'Agde. Rien ne laissait supposer qu’une autre catégorie d'internés allait rapidement les remplacer.

Avec l'entrée en guerre de la France contre l'Allemagne en septembre 1939, les conditions changèrent brutalement pour les Juifs allemands réfugiés dans l'Hexagone. Puisqu'ils étaient ressortissants d'une puissance ennemie, ils furen immédiatement considérés comme suspects, bien quétant eux-mêmes opposés au régime honni qu' ils avaient fui. La France refusa de reconnaitre que ces personnes étaient de farouches opposantes au nazisme et la manifestation de leurs opinion. antifascistes ne fut même pas prise en compte. La loi de novembre 1939 permit au gouvernement français de les placer dans des camps d'internement sans le moindre jugement et sans la moindre condamnation à leur égard. Comme Peschanski le souligne fort justement, il y avait là une remarquable absurdité d'une situation où les ennemis les plus irréductibles du nazisme se voyaient internés par la France, tout simplement parce que cette dernière avait déclaré la guerre à Hitler (Peschanski, 2008, p. 77)

Dès septembre 1939, 15000 Juifs d’Alsace-Lorraine préférèrent quitter leur région natale pour se répartir dans le Sud de la France. Puis, la Blitzkrieg de mai 1940 région natale pour se répartir dans le Sud dela France. Puis,la Blitzkrieg de mai 1940 engendra l'exode de 40000 Juifs de Belgique, du Luxembourget des Pays-Bas fuyant eux aussi vers le Sud. La débâcle générale et l'effondrement de l'armée française entraînèrent la fin de la Troisième République. Le 17 juin 1940, le maréchal Pétain dernier Président du Conseil de cette Troisième République, demandait l'armistice. L'armistice du 22 juin 1940 divisait principalement la France en deux zones séparées par une ligne de démarcation courant sur $1100 \mathrm{~km}$ de l'Espagne atlantique à la Suisse : au Nord, la zone occupée par l'armée allemande; au Sud, la zone libre sous la seule souveraineté du gouvernement de Vichy. Conséquemment, cette zone libre apparut immédiatement comme un havre de sécurité etcomme une route de survie pour les réfugiés juifs qui y accoururent de partout (Alary, 2001, 2003).

À partir de l'instauration de l'État français le 10 juillet 1940, la population juive, de nationalité française et de nationalité étrangère, fut incorporée sous le vocable de l'anti-France. Aux yeux des dirigeants de l'État français, l'anti-France, c'était les communistes, les socialistes, les anarchistes, les Tsiganes, les immigrés, les anciens des Brigades internationales, les Républicains espagnols, les francs-maçons, les résistants gaullistes, les sympathisants de la Grande-Bretagne en lutte et, d’abord et avant tout, les Juifs. Il est un aspect capital qu'il faut bien garder à l'esprit : la législation anti-juive de Vichy ne fut aucunement imposée par les Allemands. C'est ains que fut institué le Statut des Juifs le 3 octobre 1940. Certains ont pu dire à l'époque que ce statut était un scandale pour la conscience chrétienne et un défi à l'intellidé sur la discrimination raciale (Grynberg, 1999). Puis, dans la foulée immédiate de ce statut, un décret du
4 octobre 1940 permit d'interner les Juifs non français dans des camps d'étrangers, notammentà Gurs, au Vernet d'Ariège, à Rivesaltes, à Agde et aux Milles (Fredj, 1996).

À la fin de l'année 1940, 28000 Juifs étaient ainsi internés dans les camps de a zone libre. Ils passèrent à 40000 en février 1941 pour retomber à 9800 début août 942 (Grynberg, 1999). En mars 1941 était institué un Commissariat aux Questions Juives. Le second Statut des Juifs (juin 1941) aggrava encore la situation de cette population. C'́tait la mise au ban d'une partie de la communauté francise. cette pepse Bref, il etait clair quála fin 1940 et au début 1941 deux types de populations juives se trouvaient concentrées dans la zone libre dite zone non occupée, issue de l'Armistice. D’une part, il y avait les Juifs étrangers qui étaient internés dans les camps de Vichy. D’autre part, il y avait les Juifs français échappés de la zone occupée. Bien que recensés et surveillés, ils étaient libres de leurs mouvements et ne portaient pas létoile jaune, à l'inverse de la zone occupée. En 1941, on recensait environ 110000 Juifs en zone libre (Poznanski, 2005).

\section{LES JUIFS ÉTRANGERS DANS LES CAMPS DE LA ZONE LIBRE}

Avant d'aborder la géographie des cinq camps concernés par cette étude, il convient tout d'abord de bien saisir leur contexte spécifique. Dès l'automne 1940 convient tout d'abord de bien saisir leur contexte spécifique. Dess l'automne 1940, les Républicains espagnols furent remplacés dans ces camps par des Juifs étrangers en vertu du décret du 4 octobre 1940 déjà mentionné. Ainsi en novembre 1940, 26000 étrangers internés étaient dénombrés dans ces camps. Poznanski a retracé la répartition juive dans ces camps à lété 1941 : les Juifs constituaient $90 \%$ de la population internée du camp de Gurs, $80 \%$ de celle des Milles, $40 \%$ de celle de Rivesaltes et $20 \%$ de celle du Vernet (Poznanski, 2005, p. 223). En novembre 1940 se déroula une opération exceptionnelle, restée longtemps inconnue dans l'histoire de la Shoah. Voulant rendre l'Allemagne judenrein (vide de Juifs) et alors que la Solution finale n’avait pas encore été planifiée, les diriceants nazis décidèrent d'expulser en zone libre des Juifs allemands originaires du Pays de Bade, du Palatinatet de la Sarre. zone libre des Juifs aller (Went au camp de Gurs pour 6538 d'entre eux et à Rivesaltes pour les 1125 autres (Wiehn, 2010).

\section{Le camp de Gurs}

Installé sur une terrasse alluviale dominant la vallée du Gave d'Oloron entre Navarrenx et Oloron Sainte-Marie (Pyrenees-Atlantiques), le camp de Gurs fut construit en quarante-deux jours en mars-avril 1939 sur le territoire de trois communes : Gurs, Dognen, Préchacq-Josbaig. Il reçut d’abord des Républicains espagnols. De part et d'autre d'une route asphaltée longue de deux kilomètres, les 79 hectares du camp furent divisés en 13 îlots pour un total de 428 baraques, le tout entouréde 250 lom entouré de 250 kilomètres de barbelés. Plus précisément, lenceinte du camp mesu- 


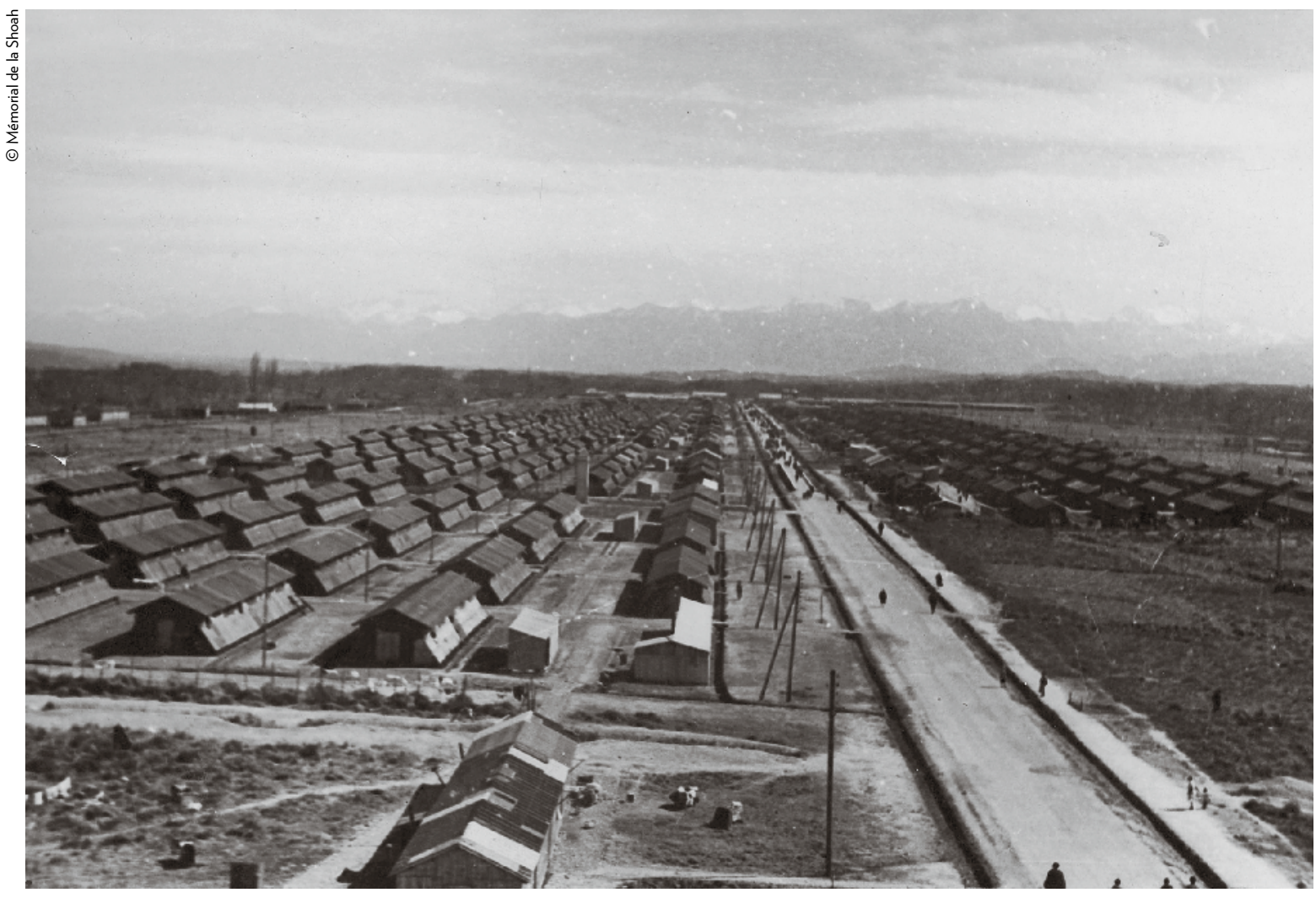

- Le camp de Gurs.

18500 individus. En mai 1940, la population internée à Gurs s’élevait à 12000 personnes. Parmi les internées juives, on retrouvait la philosophe Hannah Arendt (1906-1975) et las internés juives, on retrouvait la philosophe Hannah Arend 59). Le 24 octobre 1940 les 6538 Juifs originaires du Pays de Bade, du Palatinat et de la Sarre entraient au camp. Dans les semaines suivantes, 800 d'entre eux moururent de froid, de faim et de chagrin. Ensuite, entre octobre 1940 et novembre 1942, 7010 autres Juifs arrivèrent à Gurs, transférés d'autres camps comme Les Milles, Rivesaltes, Le Vernet et d'autres (Wiehn, 2010).

Le rabbin Léo Yehuda Ansbacher (1907-1998) réussit à maintenir une vie spirituelle juive dans le camp en l'absence des livres de prières. En avril 1941, pour la fête de Pessa'h (la Pâque juive), on recopia à la main et de mémoire une édition de la Haggadah, ce livre de prières lu lors de la célébration du Seder (repas pris les deux premiers soirs de la Pâque et retraçant l'Exode hors d'Égypte du peuple hébreu). Le rabbin Ansbacher put alors célébrer Pessa'h en lisant la prière rituelle de la Haggadah
(Gutterman \& Morgenstern, 2003).
La faim, le froid, l'humidité, la vermine, les maladies, le manque d'hygiène, les mauvaises conditions de vie pesaient quotidiennement sur les internés. Berlinoise juive antinazie exilée en France, Hanna Schramm fut internée à Gurs en 1940-1941. Ultérieurement, elle écrivit le récit de son fonctionnement quotidien (Schramm \& Vormeier, 1979). Entre octobre 1940 et octobre 1943, on compta 1038 décès à Gurs, ce qui représente un taux de mortalité énorme, rapporté à la population totale du camp. Le pire fut la boue. Le camp de Gurs était construit sur un plateau argileux inapte à la culture et mal drainé Gurs fut toujours un immense bourbier (Laharie, 2005). Le camp fut officiellement dissous le $\mathrm{l}^{\text {er }}$ novembre 1943.

\section{Le camp du Vernet d'Ariège}

Localisé dans la vallée de l'Ariège sur la commune de Saverdun, entre Saverdun au Nord et Pamiers au Sud, le camp du Vernet s'étendait sur cinquante hectares divisés en trois sections, séparées les unes des autres par des barbelés et par des tranchées. Le camp lui-même était entouré d’une triple rangée de barbelés (Grynberg, 1999, p. 69). Ce camp avait été construit à partir de juin 1918 pour recevoir des prisonniers allemands et autrichiens. En 1939 et 1940, il servit de lieu d'internement pour les Républicains Rép Arthur Konditions (lie dela tere (Koestler, 1946). Cest inadaptees àlhiver, dans son livre La lie de la terre (Koestre, 1946). Cest au Vernet qu'il composa l'essentiel du célèbre Le zéro et l'infini, premier grand livre à avoir analysé les mécanismes du stalinisme (Delpla, 1994, p. 50). Dès septembre 1939, Le Vernet accueillit les Juifs étrangers et devint un camp répressif pour étrangers suspects (Cohen \& Malo, 1994). En octobre 1941, on y comptait 2000 internés. Dans le cadre des grandes rafles lancées par Vichy contre les Juifs de la zone libre en août 1942, 1500 d'entre eux arrivèrent au Vernet, majoritairement des femmes et des enfants. En septembre 1942, une quarantaine d'enfants juifs du camp étaient déportés vers Auschwitz. Le camp ferma ses portes en juin 1944, quelques jours

\section{Le camp de Rivesaltes}

Le camp de Rivesaltes est le plus connu de tous les camps d'internement juifs mis en place par Vichy en zone libre pour au moins deux raisons. D'une part, c'est le camp d'où le plus grand nombre de Juifs fut envoyé à Auschwitz. D'autre part, c'est le camp pour lequel le plus grand nombre d'études et de témoignages a été publié sous forme de livres. En 1938 fut donc construit à Rivesaltes le camp Joffre (du nom du Maréchal Joffre, natifde Rivesaltes) pour des usages militaires. Tout commeGurs et Le Vernet il servit en 1939-1940 de lieu d'internement pour les Républicains espagnols de la Retirada A quelques kilomis de la Retirada. $\mathrm{A}$ gl 
large espace plat ouvert à toutes les intempéries méditerranéennes, dont la terrible tramontane, et soumis à la chaleur écrasante de lété (Marcos \& Marcos, 2009). Le camp couvre 612 hectares, dont 13 hectares bâtis, étalés sur 4 kilomètres de long et 2 kilomètres de large pour un périmètre total de 7 kilomètres. Le plan est tracé au cordeau avec des îlots contenant chacun 70 baraques blanches en fibrociment, aux toits en tuiles plates légères. Chaque baraque fait trente mètres de long, cinq mètres de large et trois mètres de haut. En 1942, Rivesaltes devint le Centre National de Rassemblement des Irrálites etconstitua l'́lément central du réseau des camps de Rassemblement des Israelltes et constitua lelém la zone libre. En octobre 1940, on y comptait envis du Reich. En janvier 1941, on dénombrait 1916 internes puis 6644 en septembre 1941, 3478 en février 1942 et 7000 en aout 1942 au moment des rafles et des envoi vers Auschwitz via Drancy. En juillet 1942, tous les Juifs internés dans les camps de Gurs, du Vernet et des Milles étaient transférés et rassemblés à Rivesaltes. La faim, la peur, le malheur s'y exprimaient en yiddish, en allemand et en polonais. Les conditions sanitaires y étaient déplorables si bien que la maladie, accentuée par le froid et la pénurie alimentaire, fit monter le taux de mortalité à vingt pour mille. La situation climatique particulière du camp et l'aspect lugubre du lieu n'on fait qu'ajouter aux souffrances physiques et à la détresse morale des internés (Mettay, 2008). Selon les calculs de Boitel, 21283 personnes passìnt dans le camp (e camp entre le 14 Javergement et le 24 novembre 1942, date de safermeture par Vichy (Boitel, 2001). La presse suisse et la presse américaine dénoncèrent, en leur temps, les conditions d'internement à Rivesaltes. Les femmes, les hommes et les enfants d'une même famille étaient séparés dans des îlots différents. Or, ces îlots pouvaient être éloignés de plusieurs centaines de mètres, tout en étant clôturés de barbelés. Ces Juifs étrangers internés à Rivesaltes étaient soit des Allemands, soit des Polonais, soit des ressortissants de la Ville libre de Dantzig, soit des Soviétiques, soit encore des citoyens d'autres pays de l'Europe centrale. Entre 1941 et 1942, le nombre d'évasions à Rivesaltes séleva à 1832 cas. L'invasion de la zone libre par la Wehrmacht le ll novembre 1942 mit fin à l’internement des Juifs à Rivesaltes.

\section{Le camp d'Agde}

Ce camp est abordé dans cette étude dans la mesure où il fit partie du réseau des

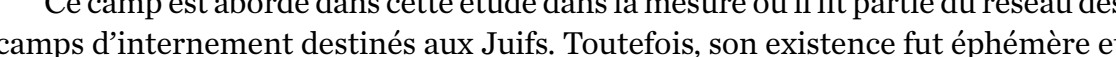
camps d'internement destinés aux Juifs. Toutefois, son existence fut éphémère et sa population juive internée fut transférée à Rivesaltes en 1942. Le camp d'Agde fut créé en février 1939 pour recevoir les Républicains espagnols. Construit en baraques légères comme à Gurs, il était localisé en sortie de la commune d'Agde sur la route de Sète. Prévu pour recevoir 20000 personnes, il accueillit finalement 24000 Espagnols dès le 15 mai 1939. Le camp était vide à la fin août 1940, mais en décembre 1940, il abritait de nouveau 500 pers toun abritá los femmes, d'un côté, les fence et les pents, de

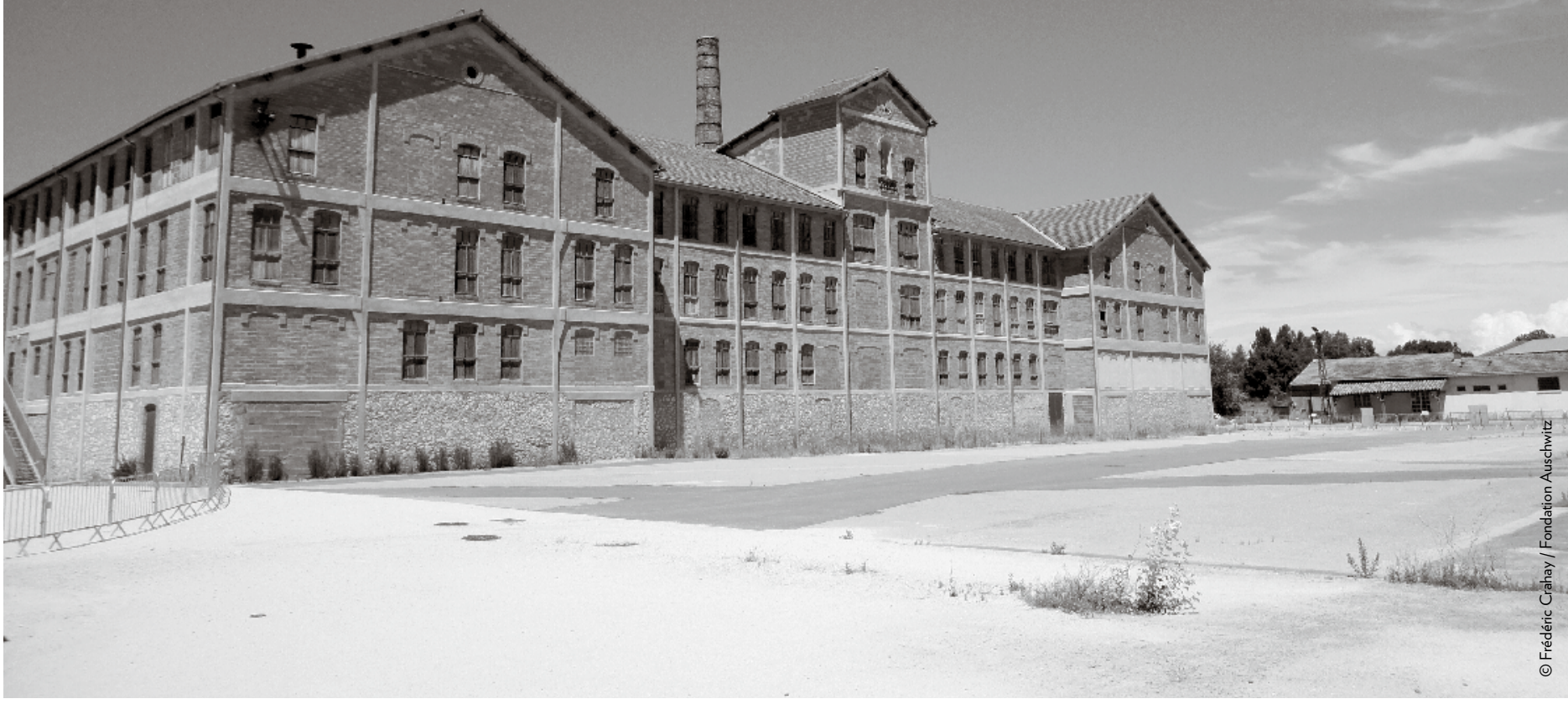
déplorables, car les infrastructures trop légères avaient vieilli. En outre,l'hiver yétait
particulièrement rigoureux. En janvier-février 1941, des convois de Juifs polonais quittèrent le camp pour Rivesaltes. La rafle du 26 août 1942, organisée par Vichy dans quittèrent le camp pour Rivesaltes. La rafle du 26 août 1942, organisée par Vichy dans Le camp fut démantelé à l'automne 1943 et détruit en août 1944 (Dauphin, 2006).

\section{Le camp des Milles (Aix-en-Provence)}

En périphérie du village des Milles, lui-même situé à 5 kilomètres au Sud du centre historique d'Aix-en-Provence (et annexé postérieurement à cette ville), le camp fut installé dans une tuilerie en faillite. Début septembre 1939 l'armée déblaya les locaux et entoura l'usine avec des barbelés afin d'y interner les ressortislaya (l) ) ou encore recrivain Lion Feuch antifasciste Alfred Kantorowicz (1899-1979) ou encore lecrivain Lion Feuchtwanger (1884-1958). Le premier livrera ultérieurement ses souvenirs du camp dans Exil in Frankreich. Merkwürdigkeiten und Denkwürdigkeiten (Kantorowicz, 1986). Le second retracera la vie du camp dans son livre Le Diable en France (Feuchtwanger, 2010). La majorité de ces internés était juive. Le bâtiment principal se présentait sur trois étages pour une superficie de $25000 \mathrm{~m}^{2}$ ouvrant sur une cour de $45000 \mathrm{~m}^{2}$ (Fontaine, 1992, p. 14-15). Tout comme à Rivesaltes et à Agde, les conditions climatiques méditerranéennes étaient omniprésentes :la cour était fréquemment balayée par le mistral et envahie de poussière. Mêmes conditions sanitaires exécrables que dans les autres camps la pille, les poux, les punaises, les puces dè juin 1940, le dans les át res camp comptait 3500 internés. Pendant tout le mois daoût 1942, il y fut procédé à
- Le camp des Milles-
juin 2014. 
199). En septembre 1942, le camp était vide. Après l'invasion de la zone libre par la Wehrmacht le 11 novembre 1942, il fut transformé en dépôt de munitions.

\section{LANTICHAMBRE DE LA SHOAH : UN ITINÉRAIRE VERS AUSCHWITZ}

La convention d'armistice du 25 juin 1940 entre l'Allemagne et la France créant une ligne de démarcation séparant la zone occupée de la zone libre instaura une dichotomie du territoire français qui eut des conséquences immédiates sur le sort dichotes sur le sort des Juifs. En clair, jusqu'au 11 novembre 1942, date de l'invasion de la zone libre par la Wehrmacht suite au débarquement anglo-américain en Afrique du Nord, Vichy eut la pleine maitrise de la situation des Juifs en zone libre. Dans un premie temps, de l'automne 1940 à juin 1942, les Juifs étrangers furent internés dans les camps de la zone libre. La situation changea brutalement lorsque Pierre Laval, chef du gouvernement de Vichy, accepta, le 4 juillet 1942, de livrer au pouvoir nazi les Juifs étrangers de la zone libre. Ce processus s’était mis progressivement en place depuis l'accord Oberg-Bousquet du 16 juin 1942 prévoyant la livraison de 10000 Juifs étrangers de la zone libre qui seraient arrêtés par la police française puis embarqués dans des convois de déportation vers Drancy, la salle d'attente d'Auschwitz Ces mesures brutal s'attirent sur une communauté juive qui avit fait confance a mestes ance à la France, mais qui était interne depuis des condition de très grande détresse morale : privation de liberté, règles administratives draconiennes, promiscuité permanente, inconfort généralisé, sous-nutrition, insalubrité angoisse (Grynberg, 1999). Toutefois, jusquà l'été 1942, et malgré ce contexte difficile, les Juifs étrangers internés n'avaient pas le sentiment qưon les emmenait à la mort. Certes, ils ne croyaient plus en la France mais ils souhaitaient, à tout le moins, émigrer vers un pays d'outre-mer ou non belligérant (Mexique, Palestine britannique, Etats-Unis, Suisse).

La radio anglaise, captée dans certains camps, avait parlé de déportation prochaine en Pologne sans autre précision. Pour ceux et celles dans les camps qui chaine croyaient à cette idee, il ne pouvait s'agir que d'un transfert dans un camp de travail à la dure discipline et situé dans un pays au climat rigoureux. Personne ne voulai croire à la Solution finale pourtant adoptée dès la Conférence de Wannsee du 20 janvier 1942. Le 18 août 1942, le gouvernement de Vichy prépara les aspects concrets de la grande rafle du 26 août 1942 ayant pour objectif l'arrestation de 15000 Juifs étrangers de la zone libre et leur envoi à Drancy. Toutefois, cette rafle ne donna pas les résultats escomptés puisque 6584 personnes seulement furent arrêtées. La déportation vers Auschwitz prit des itinéraires quelque peu différents suivant la localisation des camps et leur proximité des voies ferrées (Grynberg, 1999).

À Gurs, d'août 1942 à mars 1943, ce sont 3907 hommes, femmes et adolescents qui furent déportés vers Auschwitz en passant par le camp de transit de Drancy. Cela se réalisa en six vagues de déportation qui vidèrent les îlots de leurs occupants à savoir les 6, 8, 24 août et $1^{\text {er }}$ septembre 1942, les 27 février et 3 mars 1943 (Kapel, 1994 ; Laharie, 2005). Le premier convoi du 6 août 1942, le plus important de tous, concerna 1700 internés. Siegbert Plastereck, lui-même internéà Gurs, en fut témoin "Qui oubliera les longues colonnes traversant le camp, silencieusement, hommes et femmes, et laissant derrière eux un vide dans lequel on croyait étouffer? » (Laharie, 1994, p. 115)

Puis un autre grand convoi eut lieu le $1^{\text {er }}$ septembre 1942. Les déportés furent rassemblés à l'entrée du camp, avant d'être embarqués dans des camions à destination de la gare d'Oloron Sainte-Marie. Jeanne Merle d'Aubigné, responsable de la CIMADE protestante à Gurs, témoigna :

Ils étaient là, assis par terre ou sur leur pauvre baluchon, consternés, affaissés, immobiles. Ils semblaient avoir perdu toutes leurs forces, toute possibilité de s'exprimer.Quelques-uns avaient l'air déjà morts, d'autres avaient un faciès d’agonisant. Je cherchais des figures de connaissance. Beaucoup étaient devenus méconnaissables en quelques heures. Dans le fond, je reconnus deux silhouettes droites, impeccables dans leur uniforme d'infirmière, l'insigne juif bien en vue. Je leur dis mon admiration de les voir ainsi. Elles répondirent

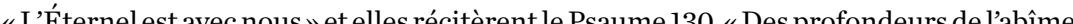

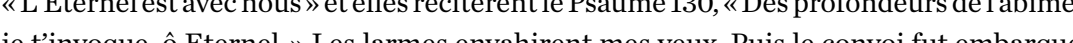
is le convoi fut embarque (Laharie, 1994, p. 116).

Au camp de Rivesaltes, une partie des Juifs étrangers arrêtés en zone libre lors de la grande rafle du 26 août 1942 y fut internée, soit environ 4538 personnes. Elle vint s’ajouter aux 1176 Juifs déjà enfermés dans le camp. Si Klarsfeld a dit de Rivesaltes qu'il fut le Drancy de la zone libre, c'est parce que neuf convois contenant au total 2313 Juifs furent dirigés vers Auschwitz via Drancy (Klarsfeld, 1993). Ces convois partirent de Rivesaltes les 11 et 19 août, les ${ }^{\text {er }}$, 4, 13, 21 et 28 septembre, les 5 et 20 octobre 1942 (Boitel, 2001). Friedel Bohny-Reiter, infirmière suisse au camp, déclarée ultérieurement Juste parmi les Nations, en fit le récit:

19 août 1942. Chaleur accablante sur le camp. Le fil de fer barbelé tiré étroitement autour des îlots $\mathrm{K}$ et $\mathrm{F}$ est oppressant. Les plaintes des gens tourmentés flottent encore dans l'air. Je les vois partir en longues files de leurs baraques haletant sous le poids de leurs affaires. Les gardiens à leurs côtés. Se mettre en rang pour l'appel. Attendre des heures dans un champ exposé au soleil. Puis arrivent les camions qui les mènent vers les voies de chemin de fer. Ils sortent des camions entre deux rangées de gardiens, les uns hésitants, les autres apathiques, quelques-uns d'un air défiant, la tête haute, dans les wagons à bestiaux. Cela dure des heures jusquà ce que tous soient entassés dans les wagons où il fait une chaleur étouffante. Je vois des visages connus à travers les barreaux. Formulant en

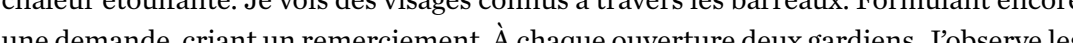
une do Du dernier wagon, on entend un au revoir (Bohny-Reiter, 2010, p. 151-152). 
13 septembre 1942. Une femme de confession protestante de Belgique qui était venue ici avec ses deux enfants pour y chercher son mari a été saisie pour remplacer la dernière personne manquante. Ses cris dans la nuit étaient terribles. Avant d’arriver au wagon de chemin de fer, elle s'est cramponnée aux gardiens. Cela ne lui a servi à rien. La porte en fer s'est fermée sur elle, et on n'entendait plus que ses pleurs à travers les barreaux de fer accusant l'humanité dans sa totalité (Bohny-Reiter, 2010, p. 161-162).

Au camp des Milles, les trains envoyèrent à Auschwitz via Drancy un total de 1928 Juifs en août et en septembre 1942. Raymond-Raoul Lambert (1894-1943) 1928 Juifs en août et en septembre 1942. Raymond-Raoul Lambert (1894-1943) était le directeur général de l'Union Générale des Israélites de France. Il fut déporté à Auschwitz le 7 décembre 1943 avec sa femme et ses quatre enfants, tous gazés à Auschwitz le 10 décembre 1943. Dans le cadre de ses fonctions, il se trouvait aux Milles en août 1942 et raconta le départ du convoi :

Lundi 10 août : journée terrible. Quels gestes de pauvres pères qui, avant la déportation définitive, caressent le visage d'un fils ou d'une fille, comme pour en conserver l'empreinte au bout des doigts! Des mères hurlent de désespoir et personne ne peut retenir ses larmes. Certaines femmes conservent une remarquable distinction. Les wagons, noirs comme des corbillarde attendent sur la voie de garage. Encadrés par des gardes en ames, des descos, des humains qui hont comis ays qui leur avait promis asile àceux qui seront leurs bourreaux (Lembert, 1985, p. 209).

Le pasteur Henri Manen (1900-1975), aumônier du camp et déclaré ultérieurement Juste parmi les Nations, était présent au départ du convoi du 2 septembre 1942

Ce qui était particulièrement douloureux à voir cétait le spectacle des petits enfants. Car des ordres stricts furent donnés en dernière heure tels quau-dessus de deux ans, tou devaient obligatoirement partir avec leurs parents. Ceux-ci n’ayant plus le choix de les laisser derrière eux à une organisation charitable. Des enfants tout petits trébuchaient

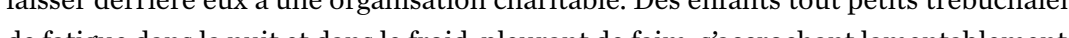
de farge dent leurspants peleurs paquets et de leurs bagages; de pauvres petits bonshommes de cinq ou six ans essayan de porter vaillamment un baluchon a leur taille puis tombant de sommeil et roulant par terre, eux et leurs paquets, tous grelottant sous la rosée de la nuit dans une attente qui s'est prolongée pour certains groupes pendant des heures; de jeunes mères et pères pleurant silencieusement et longuement dans la constatation de leur impuissance devant la souffrance de leurs enfants; puis lordre de départ donné pour quitter la cour et partir au train. Les pauvres petits tenant leur place et leur numéro dans les rangs et emboitant le pas de leurs petites jambes flageolantes (Grandjonc, 1984, p. 215).

Qu'il s’agisse des internés de Gurs comme de ceux du Vernet, de Rivesaltes ou des Milles, la majorité fut gazée dès son arrivée à Auschwitz et très peu de survivants revinrent en 1945

\section{LE SAUVETAGE DES ENFANTS JUIFS INTERNÉS : UNE ROUTE DE SURVIE}

Pour les Juifs des camps d'internement de la zone libre, le programme de sauvetage des enfants représenta la route de survie la plus productive, la plus efficace, mais aussi la plus institutionnalisée dans la mesure où elle reçut l'aval des autorités de Vichy (Kieval, 1980). Autour du sauvetage des enfants juifs s’est écrite une épopée peu connue, fruit de la volonté d'organisations humanitaires juives et chrétiennes et de bénévoles soit juifs françis, soitétrangers neutres qui risquèrent leur vie dans le bénéch l'organisation et la gestion de filières d'emigration (Zeitoun, 1989). En effet, Vichy donna son accord pour que puissent travailler vingt-cinq organisations humanitaires, nationales et internationales, religieuses et läques, regroupées en un Comité de Coordination dit Comité de Nîmes. Celles-ci apportèrent assistance et entraide aux internés et surtout à leurs enfants. On retrouvait dans cette coordination les organisations suivantes : YMCA, OSE juive (Euvre de Secours aux Enfants), CIMADE protestante, SSE (Secours Suisse aux Enfants), SSAE (Service Social d'Aide aux Émigrants), UGIF (Union Générale des Israélites de France), EIF (Éclaireurs Israélites de France), HICEM (Association d'Aide à l'Émigration Juive), JOINT (American Jewish Joint Distribution Committee), USC (Unitarian Service Committee), Amitie Chrétienne, Eglise Mennonite Américaine, American Friends Service Committee Chrétien (Quakers), Secours Catholique. Lobjectif premier de certaines de ces organisations était de faire émigrer aux États-Unis, au Venezuela et en Argentine les enfants juifs de moins de seize ans internés dans les camps, par des places réservées dans des paquebots au départ de Lisbonne. Il s'agissait donc d'obtenir l'autorisation des parents internés, de préparer les enfants à la séparation d’avec leurs parents et de procéder à toutes les démarches administratives nécessaires. Ces organisations disposaient en zone libre de toute une série de maisons d'accueil, de fermes-écoles, de colonies pour ces enfants (principalement dans le Massif Central et dans les Alpes) ainsi que du solarium marin de Palavas-les-Flots près de Montpellier. Dans ces maisons, les enfants étaient bien nourris et suivaient les cours des écoles et collèges des alentours. «Sauver les enfants» était devenu le leitmotiv d'ation et étion de toutes ces organisations courageuses, même si le convoi du 12 septembre 1942 au

La plus importante opération fut celle mise en place au camp de Rivesaltes Elle a pu être connue dans son intégralité grâce aux livres de deux témoins directs travaillant et vivant dans le camp : Vivette Samuel (1919-2006), Juive française, assistante résidente de l'OSE et Friedel Bohny-Reiter (1912-2001), citoyenne suisse, infirmière du SSE, Juste parmi les Nations (Samuel, 1995 ; Bohny-Reiter, 2010), Lorsque Vivette Samuel arriva au camp de Rivesaltes en novembre 1941, celui-ci abritait 20000 internés de toutes nationalités dont 5000 enfants de quatre à quinze ans. Au camp d'Agde, le même rôle capital de sauvetage des enfants fut endossé par ans. Au Sabine Zl tin (197-195), engace 
réussità aire sortir du camp 120 enfants juifs et 15 enfants tsiganes pour les remettre à des familles de confiance, leur sauvant ainsi la vie (Dauphin, 2006; Zeitoun, 1989) Au camp de Gurs, les enfants juifs de moins de seize ans furent également répartis dans les maisons d'accueil. En mai 1942, l'OSE estimait même que le problème des enfants dans les camps était virtuellement résolu.

Après l'invasion de la zone libre par les troupes allemandes le 11 novembre 1942 les conditions changèrent dramatiquement et les organisations humanitaires plongèrent dans la clandestinité pour maintenir ouverte aux enfants la route de la survie. En moins d'un an, le réseau de Georges Garel, président de l'OSE, finit par réussir à placer 1900 enfants juifs dans la région du Chambon-sur-Lignon et dans le Cévennes. Avec l'aide de la Croix-Rouge Internationale, des filières d'émigration des enfants juifs vers la Palestine britannique se mirent en place via l'Espagne après la traversée périlleuse des Pyrénées et un embarquement à Cadix. Devant la montée en puissance du contrôle nazi sur la zone libre après novembre 1942, les maisons d'accueil des enfants juifs exfiltrés des camps furent dissoutes et des filières clandestines de conduite jusqu’à la frontière suisse permirent à ces enfants, dont on avait changé l'identité, d'avoir la vie sauve.

Un événement tragique survint le 10 août 1942 au camp des Milles. Ce jour-là, l'OSE réussit à faire sortir du camp tous les enfants alors que leurs parents partaient en convois pour Auschwitz via Drancy. Témoignage d'un délégué de la Fédération des Sociétés Juives de France

Pendant qưon les faisait monter dans des cars avec leur mince bagage, des scènes déchirantes se sont produites. Les enfants jeunes, qui ne pouvaient comprendre les raison de cette séparation, saccrochaient à leurs parents et pleuraient. Les ainés, qui savaient combien la douleur de leurs parents était grande, tentaient de dominer leur peine et serraientles dents Les mères 1984, p. 209 ; Fontaine, 1992, p. 143)

Malgré ce drame, on peut dire que les organisations humanitaires privées, mentionnées plus haut, ont été les seules à ne pas abandonner les Juifs internés à leur sort. Elles ont tenté de les raccrocher au monde extérieur et à un avenir possible (Grynberg, 1999).

LA MÉMOIRE DES CAMPS DE LARCHIPEL DE LA HONTE, UN BILAN ACTUEL

Plus de soixante-dix ans après les faits et alors que les derniers survivants et les derniers témoins disparaissent il convient de replacer la mémoire de ce qui fut un archipeldela archipel de la honte, c'est-à-dire ce réseau des camps d'internement, dans le contexte général des Juifs en France entre 1940 et 1944.
Il y eut tout d’abord une relative passivité de la majorité de la population française a l'égard des Juifs (Klarsfeld, 2001; Marrus \& Paxton, 1981; Zuccotti, 1981). Les Français avaient à subir l'occupation, le rationnement alimentaire, l'existence de presque deux millions de prisonniers en Allemagne sans oublier le STO, mais aussi la censure dans les journaux et à la radio. Toutefois, l'existence de nombreuses organisations de secours aux Juifs et la désapprobation du statut imposé aux Juifs par cinq évêques du Sud de la France, dont Monseigneur Saliège à Toulouse, permettent de nuancer cette situation générale. L'imposition de l'étoile jaune aux Juifs de la zone occupée cette sit et les rafles de l'eté 1942 provoquèrent un revirement de l'opinion publique. On sait que, sur les 320000 Juifs présents dans l'ensemble de la France (zone occupée et zone libre), 75000 furent déportés (55000 Juifs étrangers et 25000 Juifs français). Comme on le voit, le régime de Vichy s'acharna davantage sur les Juifs étrangers (Peschanski, 2002). Le restant de la population juive de France échappa donc à la déportation. Cela put se réaliser, en partie, par le silence, la complicité ou l'aide active de Français anonymes. Il y eut aussi un élément qui joua, en partie, en faveur du sauvetage des Juifs: ce fut la zone d'occupation italienne. En effet, après l'invasion de la zone libre par l'armée allemande le 11 novembre 1942, Hitler accorda à Mussolini une zone d'occupation (c'est-à-dire les huit départements du Sud-Est mitoyens de l'Italie). Pendantles (x mois de bre 1943), la zone doccupation italienne joua le rôle de zone refuge pour les Juifs. Le diagnostic de Poznanski est le suivant: les nazis ne reussirent pas à déchirer le filet de protection que les Italiens avaient tendu dans leur zone. En outre, Vichy y fut obligé de cesser sa traque policière des Juifs sur ordre des autorités italiennes d’occupation. Conséquemment, la zone italienne permit aux réseaux de sauvetage juifs de s'organiser et de mieux fonctionner (Poznanski, 2005). À cet égard, il convient de rappeler le rôle de Monseigneur Paul Rémond, évêque de Nice, déclaré Juste parmi les Nations, dans l'organisation de filières de refuges pour les Juifs dans l'arrière-pays des Alpes Maritimes.

En cette seconde décennie du XXI ${ }^{\mathrm{e}}$ siècle, que reste-t-il de la présence de ces cinq camps dans leur environnement géographique respectif et quelles sont les politiques mémorielles qui y ont été mises en place?

\section{Le camp de Gurs}

Démantelé en 1946, il tomba peu à peu dans l’oubli. La zone occupée par le camp sest progressivement recouverte d'une forêt humide luxuriante dont les fossés de drainage n'arrivent toujours pas à absorber l'eau qui stagne sur cette terre argileuse. C'est sous ce couvert végétal, épais et touffus, constitué de chênes et de conifères élancés, qu'il faut imaginer les treize îlots et les centaines de baraques. À l'occasion du quarantième anniversaire de sa fondation en 1979, le camp de Gurs a commencé à sortir de l'indifférence. Des commémorations ont été graduellement organisées en présence d'anciens internés. Le grand cimetière juif et ses 1073 tombes est protégé 
et entretenu par les villes allemandes d'où provenaient les Juifs internés (Karlsruhe, Freiburg, Mannheim, Heidelberg, Pforzheim, Weinheim, Constance). Ces tombes sont groupées autour du monument dédié aux victimes juives. Un carré est réservé aux Républicains espagnols. En outre, un Mémorial National a été édifié à l'entrée Sud du village de Gurs. Il est l'œuvre de l'artiste israélien Dani Karavan. Inauguré en 1994, il est constitué d'une voie ferrée symbolique, de l'ossature d'une baraque et d'une dalle de béton entourée de barbelés. Dans la forêt qui recouvre désormais le

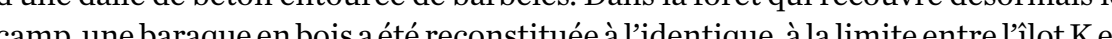
Délolîlot Ket l'îlot M. Elle jouxte l'une des tres rares fondations en beton d'une ancienne baraque, encore visible au sol. Entre le cimetière et l'emplacement de «l'îlot de représailles », on trouve la petite baraque du Secours Suisse aux Enfants où travaillait l'infirmière Elsbeth Kasser (1910-1992), surnommée «l'Ange de Gurs ». Sur la route D25 Gurs / Hôpital Saint-Blaise, là où se situait l'ancienne entrée du camp, onze colonnes de granit gris ont été installées en 2010, de part et d'autre de la route centrale du camp. Chacune porte mention de l'une ou l'autre des populations internées à Gurs (elles $\mathrm{y}$ furent très exactement 60559 ). À l'intersection entre la route express D936 Oloron-Bayonne et l'entrée Sud du village de Gurs, un bâtiment plat stylisé sert de centre d'exposition dédié à la mémoire des internés. Le site de Gurs reçoit environ 5000 visiteurs par an.

\section{Le camp du Vernet d'Ariège}

Les bâtiments du camp n'existent plus puisque les dernières baraques ont été détruites dans les années 1970. Elles ont laissé la place à un important complexe agroindustriel (silos, hangars, bureaux) et à d'immenses champs de maïs qui masquent l'essence même de ce lieu d'enfermement. À l'entrée de l'ancien camp, un Mémorial évoque ceux qui y furent internés (combattants des Brigades internationales, Républicains espagnols, Juifs étrangers). Il est placé au milieu d’un cimetière de 153 tombes d’internés. Il est reorettable que celieu de mémoirejouxte de trop orand hangars agricoles. De plus, le bruit constant engendré par l'incessant trafic routie encentie sur la route D820 désaffectée du Vernet, située le long de la voie ferrée Toulouse-Foix toujours en activité, on a placé sur des rails un wagon à bestiaux de type $\mathrm{KZ}$ identique à ceux ayant transporté les Juifs depuis la France vers les camps de la mort. Sur la façade de la gare, deux plaques commémorent le souvenir de centaines de Juifs qui furen acheminés depuis Le Vernet vers Auschwitz à partir de cette gare.

\section{Le camp de Rivesaltes}

Quand l'avion d'Air France, en provenance de Paris-Orly, commence sa descente vers l'aéroport de Perpignan, les passagers distinguent avec netteté les baraques éventrées (Mettay, 2008, p. 9). Le camp se répartit de part et d'autre de la route D5 reliant Rivesaltes à Opoul-Périllos. Les îlots J, K et $\mathrm{F}$ couvrent 42 hectares. Ils ont été achetés par le Consei Départemental des Pyrénées-Orientales pour en faire un lieu de mémoire sous la forme d'un Musée-Mémorial au coût de 23 millions d'euros. Il faut savoir que ce sont ces îlots J, K et F qui logèrent les Juifs. L'îlot F (27 hectares), avec ses 70 baraques, a été classé Monument Historique en 2000. Les travaux du Musée-Mémorial ont démarré en 2013. Il ásté inax dé en octobre 2015 par le Premarré 015 par le Premie Ministre Manue ciotti, le batiment est en partie enfoui pour faire témoigne la «memoire enfouie». Le musee retrace également l'histoire des Républicains espagnols, des Tsiganes et des Harki qui ont été internés dans le camp à des époques différentes. Cet ensemble mémoriel exceptionnel comporte une médiathèque, une salle de conférence et un centre de recherche universitaire. Des baraques ont été gardées en l'état tout autour du Musée-Mémorial qui est donc appelé à deven un espace de mémoire vivante l'importance du site, le visiteur regrettera simplement présence d'un important parc de huit éoliennes, trop visible et trop bruyant, le long de la limite sud de lîlot F Il vient perturber le recueillement et le silence indispensables à ce lieu.

\section{Le camp d'Agde}

Il fut complètement démantelé et détruit en août 1944. Aujourd'hui, il ne reste donc plus aucune trace directe du camp, mais une lecture au second degré à travers un monument une plaque de façade et trois stèles. Peu à peu, après sa destruction, la vague d'urbanisation des quartiers orientaux d'Agde a littéralement recouvert et dévoré le site. Âr monume municipalité en 1989. Sur la façade du Collège René Cassin, une plaque rappelle le souvenir des 1200 enfants juifs et tsiganes internés au camp, dont 300 de moins de trois ans. Mention est faite de l'action de sauvetage de Sabine Zlatin, la Dame d'Izieu. À l'arrière du collège, trois stèles sont dressées. La première évoque le souvenir de huit Justes agathois « de la part du peuple juif reconnaissant». La seconde est dédiée aux 41 Juifs des GTE déportés à Auschwitz le 28 août 1942. La troisième est consacrée aux martyrs des camps de Buchenwald, Struthoff, Dachau et Dora. Elle contient une urne renfermant de la terre de chacun de ces camps. Dans la ville d'Agde, aucune signalique routerren'ing camp, pas plus que la présence de ces monuments. Le contraste est frappant par rapport à la
valorisation mémorielle des camps de Gurs, de Rivesaltes et des Milles. 


\section{Le camp des Milles (Aix-en-Provence)}

Parmi les cinq camps analysés dans cette étude, celui des Milles est resté quas intact pour deux raisons très claires. D'une part, tous les autres camps avaient été édifiés très rapidement et étaient constitués de baraques de planches ou de fibrociment, légères et peu solides. Aux Milles, le camp s’était installé dans une usine à plusieurs étages, construite en briques. Il s'agissait d'une tuilerie (Fontaine, 1992). Latelier de menuiserie a été clussé Monument Historique en novembre 1993, car L'ate il renferme dout entière a té classee Mon des Milles a été inaugure en septembre 2012 par le Premier Ministre Jean-Marc Ayrault. La Fondation Mémoire du Camp des Milles espère accueillir 100000 visiteurs par an, dont 40000 scolaires. Outre lédifice proprement dit, on trouve en face de la tuilerie un wagon à bestiaux ayant servi au transport des internés. Il est place le long du quai de la voie de garage d'où partaient les convois. Sur ce quai, plusieurs stèles et plaques commémoratives évoquent le départ des internés vers les camps de la mort. Le camp des Milles présente l'avantage d'être situé dans le tissu urbain de la ville d'Aix-en-Provence, c'est-à-dire de disposer d'un accès facile, y compri par les transports publics.

En létat actuel de la question, il apparaît clairement que la survie matérielle de ces sites particuliers demande la mise en œuvre de gros moyens budgétaires pour la préservation de la mémoire et l'éducation des générations futures. Le Musée-Mémorial de Rivesaltes et le Site-Mémorial des Milles représentent les exemples les plus aboutis. En effet, ils sont le fruit d'une volonté délibérée d'élus politiques locaux qui ont su engendrer une dynamique financière et culturelle pour la réalisation de ces lieux de mémoire à destination du public le plus large. L'étalement urbain à Agde et l'étalement agro-industriel au Vernet d'Ariège rendent difficile ce même genre de réalisation, d'autant qu'il ne reste plus aucun élément matériel permettant aux visiteurs de visuliser ces camps et de s'y recueillir. Reconstruire les baraques des internés reviend internés reviendrait à légitime a doute qu'un meilleur état des lieux a permis une solution mémorielle plus facile à Rivesaltes et aux Milles. À cause dune reconquête forestière considérable, le camp de Gurs montre la limite de ce qu'il est possible de faire en matière de reconstitution Le paysage d'aujourd'hui n'est plus celui de l'époque alors qu'il a été préservé aux Milles et à Rivesaltes.

Finalement, une question centrale se pose. On peut se demander s'il est bon que les initiatives mémorielles et muséales soient totalement laissées au bon vouloi et au jugement des collectivités locales, qui décrètent économiquement valable l'existence d'un tourisme de mémoire ou, au contraire, le rejettent par peur de nuire à l'image locale.
BIBLIOGRAPHIE

- Alary, Eric (2001): « Les Juifs et la ligne de démarcation », in Les Cahiers
de la Shoah, n॰ 5 , p. 13-49.

- Alary, Eric (2003) : La ligne de démarcation 1940-1944, Paris, Perrin.

- Bohny-Reiter, Friedel (2010): Journal de Rivesaltes 1941-1942, Genève. - Boitel, Anne (2001): Le camp de Rivesaltes 1941-1942, Perpignan,
Presses Universitaires de Perpignan.

- Cohen, Monique-Lise \& Eric Malo (1994) : Les camps du Sud-Ouest de
la France 1939-1944. Exclusion, internement, déportation, Toulouse, Privat. - Dauphin, Irène (2006): : \& Le camp d'Agde 1939-19431, in Jean Sagnes
(dir), Agde 2600 ans d'histoire, Toulouse, Privat, p. 118-119. - Delpla, Claude (1994) : «Le camp du Vernet d'Ariège 1939-1944 w, in
Monique-Lise Cohen \& Eric Malo (dir.), op. cit.. p. 43-59.

- Feuchtwanger, Lion (2010) : Le diable en France, Paris, Belfond. - Fontaine, André (1992): Un camp de concentration à Aix-en-Provence? - Fredj, Jacques (1996) : Linternement des Juifs sous Vichy, Paris, CDJC. - Grandjonc, Jacques (dir). (1984) : Les camps en Provence. Exil,
internement, déportation, 1933-1944, Aix-en-Provence, Alinéa.

- Grynberg, Anne (1999) : Les camps de la honte. Les internés juifs des - Gutterman, Bella \& Naomi Morgenstern (2003): The Gurs Haggadah. Passover in Perdition, Jerusalem, Devora

Kantorowicz, Alfred (1986): Exil in Frankreich. Merkwürdigkeiten und
Dentwüurddigketen, Franfurt, Fischer. - Kapel, René (1994) : « Le terrible été 1942 : arrestations et déportations
massives des internés de Gurs », in Monique-Lise Cohen \& Eric Malo massives des internés de
(dir.), op. cit., p. 181-192.

- Kieval, Hillel (1980) : Legality and Resistance in Vichy France: The
Rescue of Jewish Children, New York, Proceedings of the American Rescue of Jewish Childgil
Philosophical Society.

- Klarsfeld, Serge (1993) : Le transfert des Juifs du camp de Rivesaltes

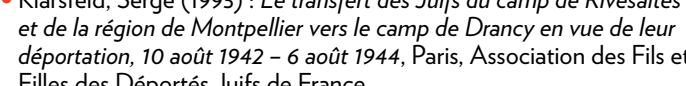
Filles des Déportés Juifs de France. - Klarffeld, Serge (2001): Vichy-Auschwitz: I e rôle de Vichy dans la
solution finale de la question juive en France, Paris, Fayard. Koestler, Arthur (1946) : La lie de la terre, Paris, Charlot.

Laharie, Claude, (1994) : « La déportation et linternement au camp de Gurs des 6538 Juifs allemands originaires du Pays de Bade et du
Palatinat (1940-1943) , in Monique-Lise Cohen \& Eric MALO (dir.), op

Laharie, Claude (2005): Gurs : 1939-1945. Un camp dinternement en
Béarn, Biarritz, Atlantica.

- Lambert, Raymond-Raoul (1985): Carnets dun témoin, Paris, Fayard. Marcos, Violette \& Juanito Marcos (2009) : Les camps de Rivesaltes Uarcos, Violette \& Juanito Marcos (2009) : Les camps de Rivesalt
Une histoire de lenfermement (1935-2007), Portet-sur-Garonne,
Nouvelles Editions Loubatières.

- Marrus, Michael \& Robert Paxton (1981) : Vichy et les Juifs, Paris Mettay, Joël (2008) : L'árchipel du mépris. Histoire du camp de Rivesaltes
de 1939 à nos jours, Canet, Trabucaire.

Peschanski, Denis (2008) : La France des camps. Linternement 1938 -
1946. Paris, Gallimard. Poznanski, Renée (2005) : Les Juifs en France pendant la Seconde Guerre
mondiale, Paris, Hachette.

- Samuel, Vivette (1995): Sauver les enfants, Paris, Liana Lévi.

- Schramm, Hanna \& Barbara Vormeier (1979): Vivre à Gurs. Un camp de Wiehn, Erhard Roy (2010): Camp de Gurs. Zur Deportation der Jude
aus Südwestdeutschland 19:40, Konstanz, Hartung-Gorre.

- Zeitoun, Sabine (1989) : Ces enfants quill fallait sauver, Paris, Albin Zuccotti, Suzan (1981): The Holocaust, the French and the Jews, New
York, Basic Books. 